Review

\title{
Molecular Imaging Radiotherapy: Theranostics for Personalized Patient Management of Neuroendocrine Tumors (NETs)
}

\author{
Kjell Öberg ${ }^{1,2 \bowtie}$ \\ 1. Department of Medical Sciences, Uppsala University; \\ 2. Department of Endocrine Oncology, Uppsala University Hospital, Uppsala, Sweden
}

$\square$ Corresponding author: Professor Kjell Öberg, MD, Ph.D. Chairman of the Centre of Excellence Endocrine Tumors. Department of Medical Sciences, Uppsala University. SE-751 85 Uppsala, Sweden. Phone No. +46 18611 49 17. kjell.oberg@medsci.uu.se.

(c) Ivyspring International Publisher. This is an open-access article distributed under the terms of the Creative Commons License (http://creativecommons.org/ licenses/by-nc-nd/3.0/). Reproduction is permitted for personal, noncommercial use, provided that the article is in whole, unmodified, and properly cited.

Received: 2011.12.08; Accepted: 2012.02.26; Published: 2012.05.08

\begin{abstract}
Neuroendocrine tumors (NETs) possess unique features including expression of peptide hormone receptors as well as the capacity to concentrate and take up precursor forms of amines and peptides making hormones that are stored in secretory granules within the tumor cells (APUD). The expression of somatostatin receptors on tumor cells have been widely explored during the last two decades starting with "'In-DTPA-Octreotide as an imaging agent followed by ${ }^{68} \mathrm{Ga}-\mathrm{DOTATOC} / \mathrm{TATE}$ positron emission tomography scanning. The new generation of treatment includes ${ }^{90}$ Yttrium-DOTATOC/DOTATATE as well as ${ }^{177}$ Lutetium-DOTATOC/DOTATATE/DOTANOC treatment of various subtypes of NETs. The objective response rate by these types of PRRT is in the range of $30-45 \%$ objective responses with $5-10 \%$ grade 3/4 toxicity mainly hematologic and renal toxicity. The APUD mechanism is another unique feature of NETs which have generated an interest over the last two decades to develop specific tracers including ${ }^{11} \mathrm{C}-5 \mathrm{HTP},{ }^{18} \mathrm{~F}-\mathrm{DOPA}$ and "C-hydroxyefedrin. These radioactive tracers have been developed in centres with specific interest in NETs and are not available everywhere. "'In-DTPA-Octreotide is still the working horse in diagnosis and staging of metastatic NETs, but will in the future be replaced by ${ }^{68} \mathrm{Ga}$-DOTATOC/DOTATATE PET/CT scanning which provide higher sensitivity and specificity and is also more convenient for the patient because it is a one-stop-procedure. Both ${ }^{90}$ Yttrium-DOTATOC/DOTATATE as well as ${ }^{177}$ Lutetium-DOTATOC/DOTATATE are important new therapies for malignant metastatic NETs. However, the precise role in the treatment algorithm has to be determined in forthcoming randomized trials.
\end{abstract}

Key words: PRRT, theranostics, ${ }^{111}$ In-DTPA-Octreotide, ${ }^{68} \mathrm{Ga}$-DOTATOC/TATE, ${ }^{90}$ Yttrium-DOTATOC, 177Lutetium-DOTATOC/DOTATATE

\section{Background}

Neuroendocrine tumors (NETs) are a diverse group of malignancies deriving from the diffuse neuroendocrine cell system with localisation almost everywhere in the human body. The most frequent localisations are in the lung, pancreas and small intestine. However, NETs can be found in the heart, middle ear, kidneys and ovaries. The incidence of NETs has constantly increased over the last decade, being now about $6 / 100,000$ with a prevalence of $35 / 100,000$.[1] The tumors are heterogeneous in terms of clinical presentation and growth. Some of them are so called functioning tumors which means that they produce peptide or amines that can cause distinct clinical symptoms such as flushing, diarrhea, hypoglycaemia, gastric ulcers or skin rash. The largest group is however non-functioning tumors and usu- 
ally present with a large mass or bleeding and sometimes difficult to delineate from a classical cancer.[2] A unique feature of NET is the APUD characteristics which means that they concentrate and take up amino acids and precursors and produce hormones that are stored in secretory granules within the tumor cells. These hormones and amines are then released into the circulations or to adjacent or even into gut lumen to regulate the different physiological processes in the body.[3] This APUD characteristic is also the bases for the development of specific molecular imaging procedures using tracers that will accumulate on the bases of uptake in the tumor cells by the so called APUD mechanism. Another unique feature of NETs is the expression of different receptors on the tumor cells not only typical growth regulating factor receptors such as VEGF, IGF, EGF and so forth, but peptide hormone receptors such as somatostatin receptors as well as CCK receptors, GRP receptors and so forth. These receptors can be targeted with radiolabeled peptides for imaging and treatment.[4]

A theranostic system integrates some form of diagnostic testing to determine the presence of molecular targets for which a specific compound is intended. Molecular imaging serves these diagnostic functions and provides powerful means for non-invasively detecting disease. The term molecular is meant to describe active targeting of a specific mechanism important in a particular disease process. The reasons for a tremendous excitement of theranostics is its revolutionary approach that promises improved therapy selection on the bases on specific molecular features of disease, greater predictive power for adverse effects and new ways to objectively monitor therapy response.[5] Currently molecular imaging in the form of PET with ${ }^{18}$ Fluoro labelled glucose analog, ${ }^{68} \mathrm{Ga}-\mathrm{DOTA}-O c t r e o t a t e ~ a n d ~{ }^{11} \mathrm{C}-5 \mathrm{HTP}$ PET has revolutionised the clinical management in oncology and particular in the field of NETs. The diagnostic accuracy has been further refined by the technical combination with anatomic imaging in the form of PET/CT as well as most recently PET/MRI. The current paper will concentrate on molecular imaging in NETs using somatostatin receptor scintigraphy as well as PET-scanning with specific isotopes such ${ }^{68} \mathrm{Ga}$-DOTA-Octreotate, ${ }^{18}$ Fluoro-DOPA, 5-Hydroxytryptophan $\left({ }^{11} \mathrm{C}-5 \mathrm{HTP}\right)$. It will also discuss the powerful treatment of NETs with radioactive somatostatin compound such as ${ }^{111}$ In-DTPA-octreotide ${ }^{177}$ Lutetium-DOTATATE and ${ }^{90}$ Yttrium-DOTATOC for treatment of malignant NETs.

\section{Nuclear Medicine Imaging}

\section{Somatostatin Receptor Scintigraphy (SRS)}

Somatostatin receptors are G-protein coupled membrane glycoproteins at the moment five subtypes of human somatostatin receptors have been cloned.[4] The somatostatin analogs octreotide and lanreotide bind with high affinity to receptor subtypes 2 and 5 . The expression of somatostatin receptor type 2 is present in $70-90 \%$ of NETs. Therefore radioactive labelled somatostatin analogs allow the visualisation and staging of these tumors expressing somatostatin receptors 2 and 5 . The most commonly used radioligand is ${ }^{111} \mathrm{In}-\mathrm{DTPA}$-octreotide (Octreoscan) recently co-registration with $\mathrm{CT}$ has been implemented in nuclear medicine.[6-8] This new fusion technique allows better interpretation of the imaging finding with better anatomical localisation (Figure 1).

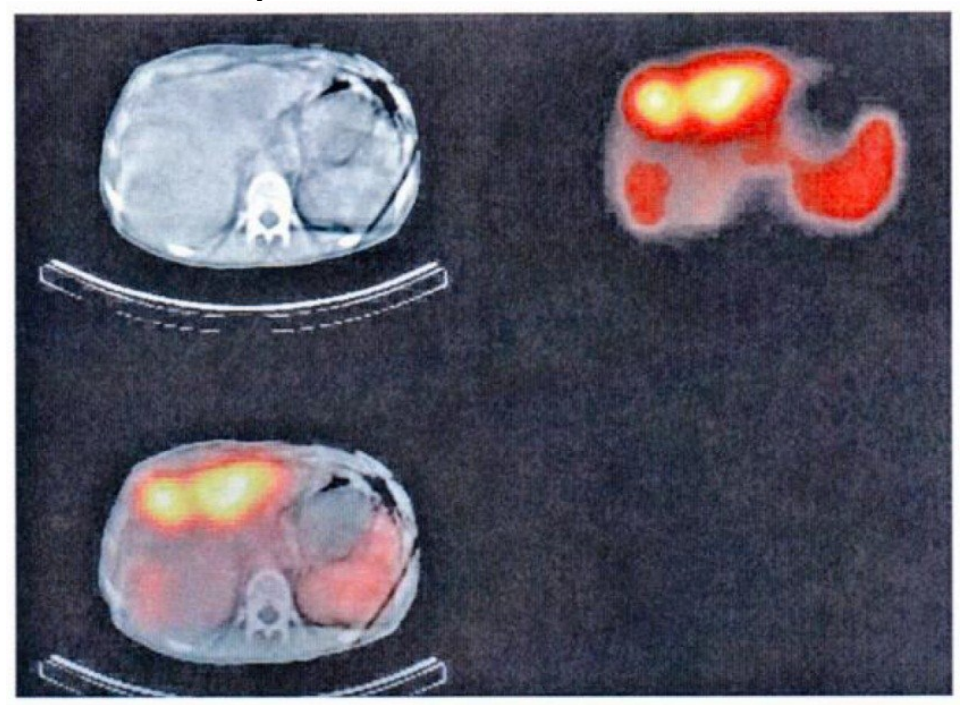

Figure I. Functioning imaging of neuroendocrine tumors using [ ${ }^{\prime \prime}$ In]DTPA-octreotide (SPECT) and CT scanning from a patient with a malignant metastatic pancreatic endocrine tumor. 
The usual dose for somatostatin receptor scintigraphy (SRS) is $200 \mathrm{MBq}$ of ${ }^{111}$ In-DTPA-octreotide. Planar images are obtained with a double headed, large field of view, gamma camera equipped with medium-energy parallel-hole collimators. If whole-body acquisition is used, the scan speed should not exceed 3-5 cm/minute. Using a higher scan speed may result in failure to recognise small somatostatin receptor positive lesions and lesions with a low density of somatostatin receptors. Single photon emission tomography-computed tomography (SPECT) may increase the sensitivity of detecting somatostatin receptor positive tissues and give a better anatomical localisation than planar views. Nowadays SPECT images are obtained using a triple-headed camera. Because of its relatively long half-life ${ }^{111}$ In-DTPA-octreotide can be used to visualise receptor-bearing tumors efficiently after 24 and 48 hours, by which times interfering background radioactivity has been reduced by renal clearance. Planar aspect studies are therefore preferably performed 24 hours after injection of the radiopharmaceutical. Repeat scintigraphy after 48 hours is especially indicated when the 24 hours image shows accumulation in the abdomen, which may represent radioactive bowel content.[8] The question arises whether somatostatin analog therapy can be administered during SRS as the uptake of ${ }^{111}$ In-DTPA-octreotide in somatostatin receptor positive tumors and the spleen will be diminished due to blocking of the receptor by 'cold' somatostatin analog. However, in our experience the majority of carcinoid tumors as well as pancreatic NETs are visualised during treatment with octreotide, although the uptake might be reduced. We therefore recommend withdrawal of the octreotide immediate-release formulation 48 hours before undertaking scintigraphy. Furthermore, during treatment with a long-acting somatostatin analog, scintigraphy should be performed just before the next injection.

The sensitivity of SRS for detecting midgut carcinoid tumors has been reported in various studies to be somewhere between $80 \%$ and $100 \%$. There is no significant difference in the results for foregut, midgut and hindgut NETs. Scintigraphy also provides a more accurate staging of disease by demonstrating tumor size that can not be shown by conventional imaging. SRS might also indicate the amount of somatostatin receptors, which may have bearing on the efficacy of treatment with somatostatin analogs (PRRT). However, it must be pointed out that there is no complete concordance between SRS results and the receptor content determined by immunohistochemistry of tumor specimens. Gastrin-producing tumors express a large number of high-affinity somatostatin receptors type 2 . The sensitivity of SRS in this case is about $80 \%$ and is the recommend staging procedure for these tumors.[9] Insulin-producing tumors, particularly the benign ones do not express somatostatin receptor type 2 and only about $50 \%$ of malignant insulin producing tumor express somatostatin receptor type 2. Other neuroendocrine pancreatic tumors, such as glucagonomas and VIPomas, express type 2 somatostatin receptors in about $60-70 \%$ of patients.[10] Thyroid medullary carcinomas and pheochromocytomas also express somatostatin receptors type 2 in about $40-60 \%$ of patients. Medullary thyroid carcinomas more often express type 1 receptors, and might therefore not be visualised by SRS. However, for both medullary thyroid carcinomas and pheochromocytomas there are other scanning procedures, such as PET-scanning, that might replace SRS. SRS is still the gold standard for diagnosis, localisation and staging of NETs.

\section{Positron emission tomography (PET)}

NETs not only express somatostatin receptors but endocrine tumors cells take up hormone precursor, express receptors and transporters and synthesize, store and release hormones.[3] Based on the so called amine precursor uptake and the decarboxylation' (APUD) concept, tracers such as ${ }^{11} \mathrm{C}$-5-hydroxytryoptophan and ${ }^{11} \mathrm{C}$-dihydroxyphenylalanine have been developed for the localisation of neuroendocrine tumors and ${ }^{18} \mathrm{~F}$ 6-fluorodopamine and ${ }^{11} \mathrm{C}$-hydroxyephedrine for the localisation of pheochromocytomas. Current tracers that are used for detection and follow-up of patients with NETs are listed in table 1.

Table I. Specific Isotopes for NETs

11C-5HTP (hydroxytryptophan)

11C-Dopamine

18F-Dopamine

68Ga-DOTA-octreotide

99Tc EDDA-HYNIC-octreotide

[Lys40(Ahx-DTPA-111In)NH2]-Exendin-4 (GLP-1)

Positron emission tomography is a non-invasive technique for measurement of regional accumulation and quantification of radioactive substances. A biological substance can be labelled with radionuclides, such as ${ }^{11} \mathrm{C},{ }^{15} \mathrm{O}$ and ${ }^{18} \mathrm{~F}$ with emission of positrons. The positrons generate photons which are detected by a special camera. The half life of standard positron 
emitters is as follows: ${ }^{18} \mathrm{~F}-110$ minutes, ${ }^{11} \mathrm{C}-20$ minutes, ${ }^{15} \mathrm{O}-2$ minutes. The most recent PET-scanners provide $2.5 \mathrm{~mm}$ slices with a resolution of approximately 5.5 $\mathrm{mm}$.

The ability to tag somatostatin analogs with ${ }^{68} \mathrm{Ga}$ has revolutionized the role of PET in diagnosis, staging and therapy monitoring of patients with receptor positive NETs. One of the advantages of the ${ }^{68} \mathrm{Ga}$-DOTANOC/DOTATOC PET or PET/CT study over ${ }^{111}$ In-Octreotide scintigraphy is better visualisation of lesions which are difficult to be seen on planar or SPECT imaging.[11-13] ${ }^{68} \mathrm{Ga}$ - DOTATOC PET appears to be superior especially in detecting small tumors or tumors bearing only low density of SRS (Figure 2). In general ${ }^{68} \mathrm{Ga}$-DOTATOC or DOTANOC PET is able to pick up many lesions which could not be picked up by CT-scan. It is of significance in therapy monitoring and is a useful adjunct in deciding the amount of radioactivity to be administered for PRRT. In an intraindividual study comparing the diagnostic efficacy of ${ }^{68} \mathrm{Ga}$-DOTANOC and ${ }^{68} \mathrm{Ga}$-DOTATATE, ${ }^{68} \mathrm{Ga}$-DOTANOC is superior to ${ }^{68} \mathrm{Ga}$-DOTATATE in terms of sensitivity.[14] In summary, among several advantages of ${ }^{68} \mathrm{Ga}$-somatostatin analogs over 111In-DTPA Octreotide scintigraphy higher sensitivity is present.[15] It is a one-stop procedure, the patient can leave the clinic after 1 hour instead of coming back 4 and 24 hours for scanning. It will also in the future be cheaper than SRS.

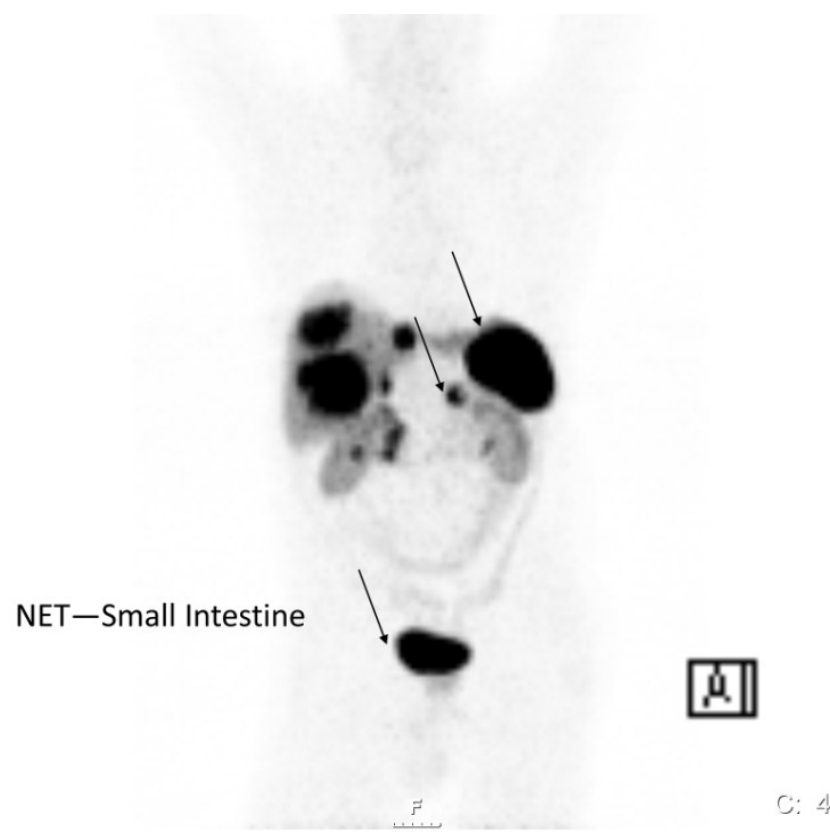

Figure 2. ${ }^{68} \mathrm{Ga}-\mathrm{DOTA}-O c t r e o t i d e ~ P E T / C T$ scanning with a patient with malignant carcinoid tumor showing multiple lymph node metastases as well as large liver metastases.
${ }^{18} \mathrm{~F}$-Fluorodeoxyglucose PET ( ${ }^{18} \mathrm{~F}-\mathrm{FDG}$ PET) is increasingly being used for various malignant tumors. ${ }^{18} \mathrm{~F}-\mathrm{FDG}$ is useful for evaluating tumor hexokinase activity, which is increased in tumor cells because of the accelerated rate of glycolysis. ${ }^{18}$ F-FDG PET is primarily used in tumors for the purpose of diagnosis, staging, restaging and evaluation of the response to treatment. The main use of ${ }^{18} \mathrm{~F}-\mathrm{FDG}-\mathrm{PET}$ in diagnosis of NETs depends on the grade of differentiation and/or aggressiveness of NETs.[16-18] ${ }^{18}$ F-FDG PET is more sensitive than SRS in picking up less differentiated GEP-tumors (G3) but is less sensitive than SRS in detection of well differentiated GEP-tumors (G1/G2) (Figure 3). In a recent study 98 prospectively enrolled patients with NETs underwent ${ }^{18} \mathrm{~F}-\mathrm{FDG}$ PET imaging.[19] The study showed a strong prognostic value of ${ }^{18} \mathrm{~F}-\mathrm{FDG}$ PET for NETs which exceeds the prognostic value of traditional markers such as Ki-67, $\mathrm{CgA}$ and liver metastases. The diagnostic sensitivity of ${ }^{18} \mathrm{~F}$-FDG PET was $58 \%$. A positive ${ }^{18} \mathrm{~F}$-FDG PET result was associated with a significantly higher risk of death with a hazard ratio (HR) of 10.3 [95\% confidence interval (CI) 1.3-78-9]. A high standard uptake value (SUV) is indicating a more aggressive disease and SUV-max of $>3$ was the only predictor of shorter progression free survival $(p=0.001)$. However, in spite of these successful results in NETs, one major limitation of ${ }^{18} \mathrm{~F}-\mathrm{FDG}$ as a tumor marker is that it is not completely specific for tumor. ${ }^{18} \mathrm{~F}-\mathrm{FDG}$ is known to accumulate in inflammatory lesions also thereby increasing the false positivity rate.[13]

NETs are known to accumulate and decarboxylate, $\quad 5^{\prime}$-hydrozytryptamine and 1-3,4-dihydroxyphenylalanine (1-DOPA). An increase in the activity of 1-DOPA decarboxylase is one of the hallmarks of NETs.[20] The first study that demonstrated the utility of ${ }^{11} \mathrm{C}$-DOPA in the detection of pancreatic tumor was conducted by our group in 1995.[21] 18F-DOPA PET has also been found to be useful in advanced NET.[22] The potential limitation of ${ }^{18} \mathrm{~F}-\mathrm{DOPA}$ PET is normally high accumulation in the duodenum and pancreas, which might cause problems in localisation of tumors in the region. Another important tracer for NETs is ${ }^{11}$ C-hydroxytroyptophan PET $\left({ }^{11} \mathrm{C}-5 \mathrm{HTP}\right.$ PET), which is based on the uptake ${ }^{11} \mathrm{C}$-hydroxytryoptophan by neuroendocrine cells, decarboxylated and then stored in vesicles as ${ }^{11} \mathrm{C}-5 \mathrm{HT}$. PET scanning with ${ }^{11} \mathrm{C}-5 \mathrm{HTP}$ visualises significantly more lesions than SRS, CT or MRI in patients with NETs.[23] The sensitivity of ${ }^{11} \mathrm{C}-5 \mathrm{HTP}$ PET is almost $90 \%$ and can detect tumors as small as $3 \mathrm{~mm}$ (Figure 4 and 5). It has been observed that ${ }^{11} \mathrm{C}-5 \mathrm{HTP}$ PET can be used in patients without elevated 5HT synthesis and it appears to be a univer- 
sal tracer for neuroendocrine tumors irrespective of origin and can visualise more than $90 \%$ of all types of NETs including endocrine pancreatic tumors.[24] Furthermore, the possibility to modulate the uptake of ${ }^{11} \mathrm{C}-5 \mathrm{HTP}$ with carbidopa has facilitated the performance of whole-body PET.[16, 23] Importantly poorly differentiated tumor with little or no hormone production (G3-tumors) can be falsely negative with
${ }^{11} \mathrm{C}-5 \mathrm{HTP}$ or ${ }^{18} \mathrm{~F}-\mathrm{DOPA}$ PET. The tracer ${ }^{11} \mathrm{C}$-hydroxyefedrin (Figure 6) has been explored in patients with pheochromocytoma. ${ }^{11} \mathrm{C}$-hydroxyefedrin has been applied in studies of pheochromocytoma presenting a sensitivity and specificity of $90-100 \%$. Other potential receptors to be targeted for diagnosis and therapy are listed in table 2.[25]

\section{Whole body FDG-PET}

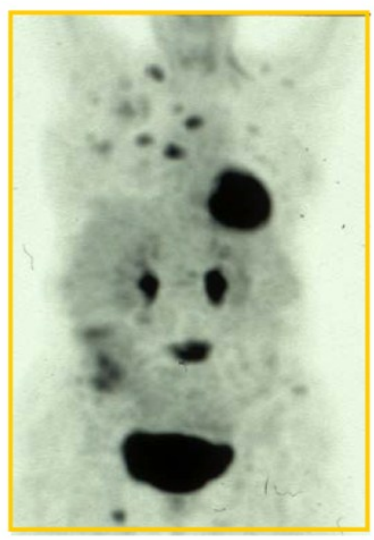

Frontal projection

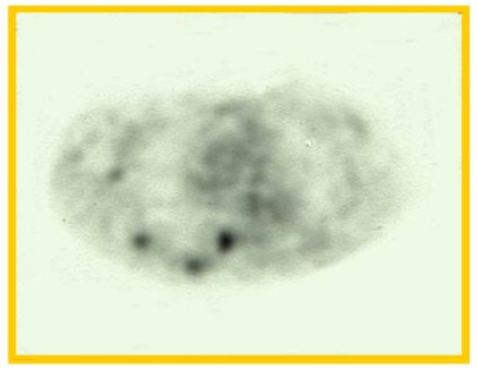

Transaxial

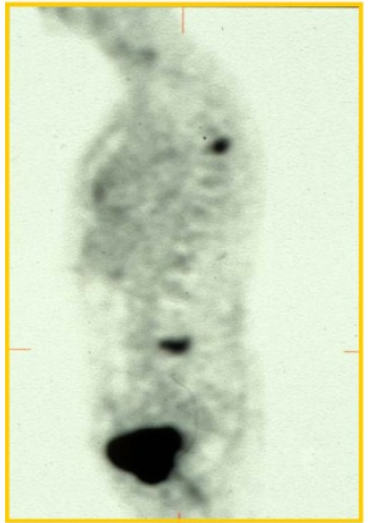

Sagittal

in a poorly differentiated neuroendocrine tumor

Figure 3. FDG/PET of a patient with a high-grade neuroendocrine tumor (G3 tumor) with bone metastases as well as lymph node and liver metastases.

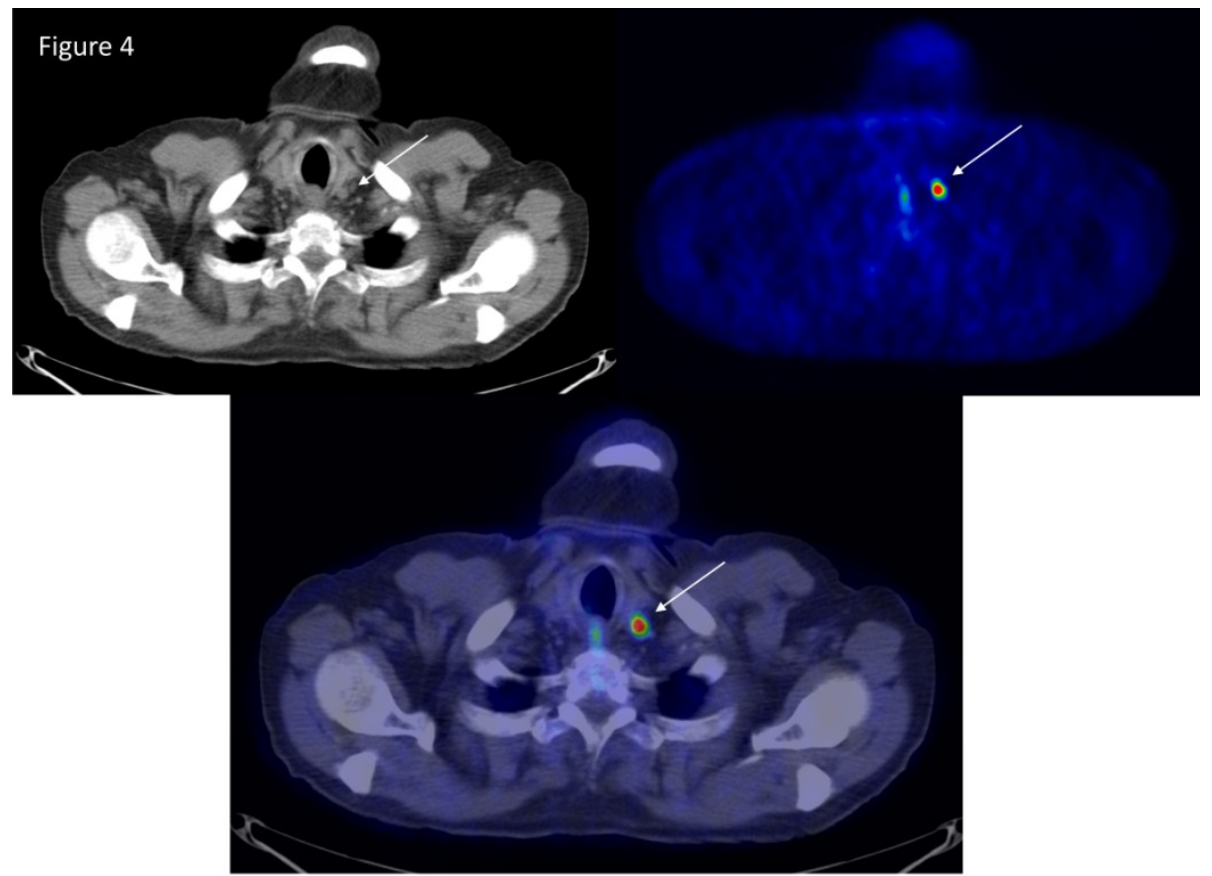

Figure 4. "C-5HTP PET/CT of a patient with a small intestinal neuroendocrine tumor (carcinoid) which was considered to be cured after surgery. A small lymph node, $<1 \mathrm{~cm}$, showed abnormal tracer accumulation demonstrating a lymph node metastasis. 


\section{PET/CT with ${ }^{11}$ C-5-HTP}

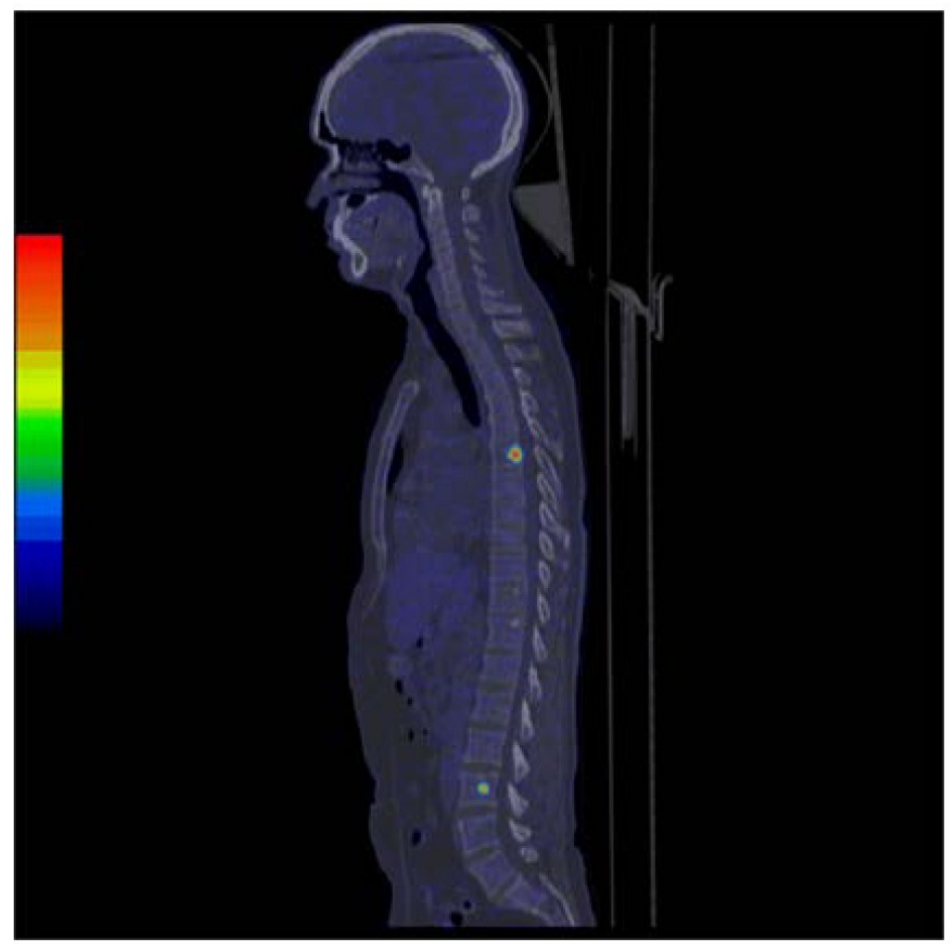

Figure 5. "C-5HTP scanning of a patient with midgut carcinoid tumor showing bone metastases that was not detected by standard radiology such as $\mathrm{CT}$ or $\mathrm{MRI}$.

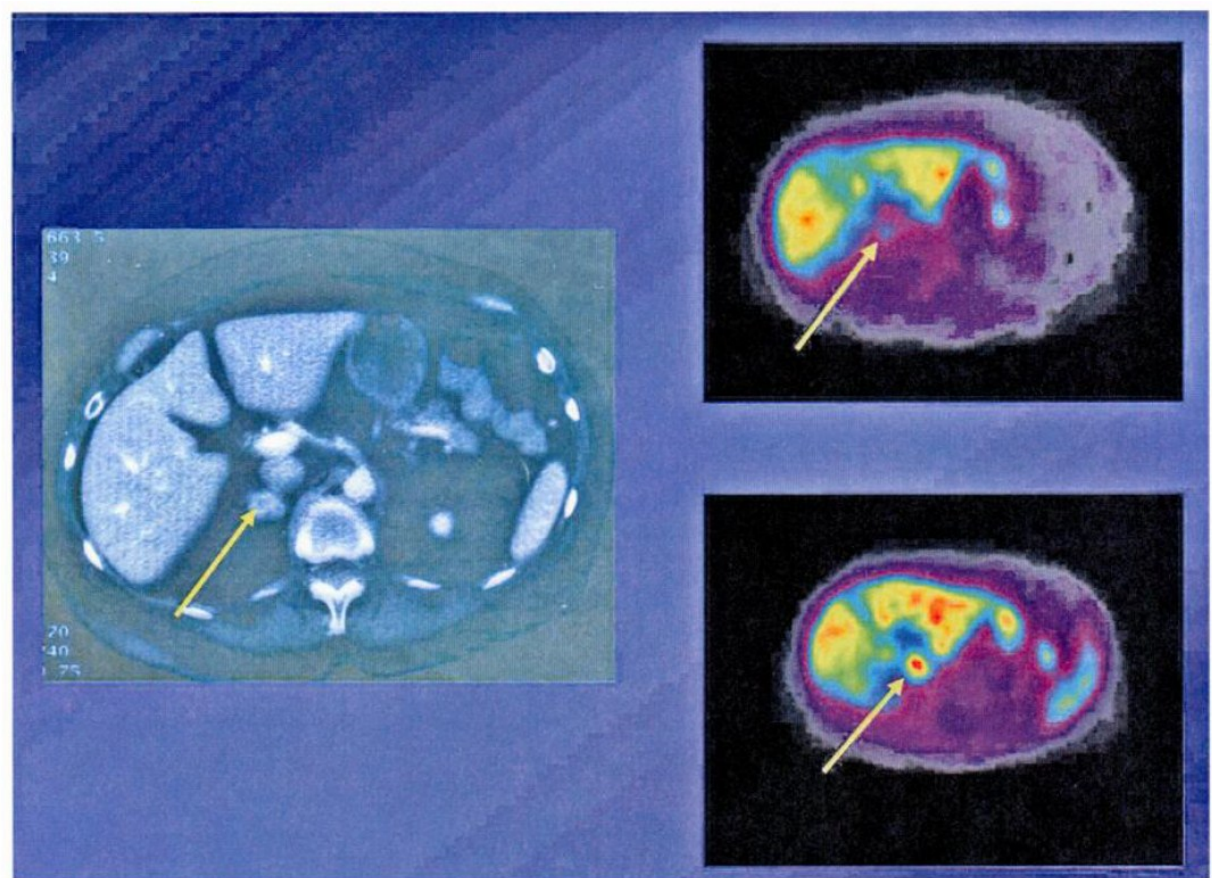

Figure 6. PET scanning with "C-metomidate and IIC-hydroxyefedrine as tracers in a patient with a $2 \mathrm{~cm}$ incidentaloma of the adrenal gland. The tumor is accumulating " C hydroxyefedrin indicating a pheochromocytoma, not an adrenocortical tumor. 
Table 2. Potential Receptors Other Than SS Receptors for Clinical Use

\begin{tabular}{|c|c|c|c|}
\hline Peptide & Receptor Subtypes & Tumor Expression & Radiolabeled Peptides \\
\hline Bombesin/GRP & GRP-R & Prostate cancer, breast cancer, GISTs, SCLC & $\begin{array}{l}\text { 99mTc-bombesin } \\
{ }^{111} \mathrm{ln} \text {-bombesin, }{ }^{68} \mathrm{Ga} \text {-bombesin }\end{array}$ \\
\hline $\mathrm{CCK} /$ gastrin & $\mathrm{CCK}_{2}$ & MTC, insulinoma, SCLC, GISTs & $\begin{array}{l}{ }^{111} \text { In-DTPA-minigastrin } \\
{ }^{99} \mathrm{Y} \text {-minigastrin }\end{array}$ \\
\hline GLP-1 & GLP-1-R & Insulinoma, gastrinoma & 123I-GLP-1 \\
\hline Neuropeptide- $Y$ & NPY-R & Breast cancer, ovarian and adrenal tumors & 99mTc-neuropeptide $\mathrm{Y}$ \\
\hline Neurotensin & NT-R1 & Exocrine pancreatic cancer, Ewing sarcoma, meningioma & 99mTc-neurotensin \\
\hline Substance P & NK1 & Glial tumors & ${ }^{90}$ Y-DOTAGA-Substance P \\
\hline VIP & $\mathrm{VPAC}_{1}$ & Gastro-intestinal and other epithelial cancers & $\begin{array}{l}\text { 123I-VIP } \\
\text { 99mTc-TP3654 }\end{array}$ \\
\hline
\end{tabular}

GRP-R, gastrin releasing peptide-receptor; CCK, cholecystokinin; GLP, glucagon-like peptide; VIP, vasoactive intestinal peptide; VPAC, VIP-pituitary adenylate cyclase-activating polypeptide; NK, neurokinin; GISTs, gastrointestinal stromal tumors.

Adapted from Rufini et al. Semin Nucl Med 36:228-247

\section{Summary}

Nuclear imaging techniques have significantly improved the localisation and staging of NETs. Besides anatomical localisation they also give information about metabolism, receptor expression and the distribution of drugs. PET imaging for tumor localisation and follow-up of treatment is more or less established in different types of endocrine tumors. In the future PET may enable the in-vivo determination of the proliferation and an alteration in gene-expression during treatment. Another advantaged of PET is the response to new therapies such as angiogenesis and tyrosine kinase inhibitors, which can be rapidly detected. Although the PET scanning is expensive it is cost effective because patients not responding to therapy do not need to continue with these expensive drugs.

\section{Peptide-receptor Radionuclide Therapy (PRRT)}

In the 1990s peptide receptor radionuclide therapy (PRRT) with radiolabeled somatostatin analogs was introduced as promising treatment modality for patients with inoperable or metastasized NETs. Given the ability of radiolabeled somatostatin analogs to identify somatostatin receptor positive tumor the next logical step was to try and use radiolabeled somatostatin analogs as a novel treatment for this group of tumors. Radiolabeled somatostatin analogs generally comprise three main parts the cyclic octapeptide (e.g.Tyr ${ }^{3}$-octreotide or $\mathrm{Tyr}^{3}$-octreotate) a chelator [e.g. DTPA or teraazacyclododecane tetraacetic acid (DOTA)] and a radioactive element. Radioisotopes commonly used in PRRT are ${ }^{111} \mathrm{In},{ }^{90} \mathrm{Y}$ and ${ }^{177} \mathrm{Lu}$.

\section{IIIIn-DTPA octreotide}

Studies in the early 1990s used the Auger-electron-emitting ${ }^{111}$ In-DTPA-octreotide for PRRT as somatostatin analogs labeled with $\beta$-emitting radionuclides were not available for clinical use at that time. Treatment with high doses of ${ }^{111}$ In-DTPA-octreotide often led to symptomatic relief in patients with metastatic NETs; However, tumor shrinkage was rarely achieved.[26-28] The most common long-term adverse effects of ${ }^{111}$ In-DTPA-octreotide therapy resulted from toxic effects to the bone marrow. Serious adverse effects included leukemia and myelodysplastic syndrome in three patients who had been treated with total cumulative doses of $>100 \mathrm{GBq}$ and in whom bone-marrow radiation doses were estimated at $>3$ Gy. Delpassand et al treated 32 patients with high doses of ${ }^{111}$ In-DTPA-octreotide, 18 of whom received two cycles of $18.5 \mathrm{GBq}$. Of the 18 patients treated with two cycles, 16 had stable disease and two had partial remission; median time to progression was not reported.[29]

\section{Y-DOTATOC}

The next generation of radionuclide therapy that targeted somatostatin receptors was the radiolabeled, modified somatostatin analog ${ }^{90} \mathrm{Y}$-DOTA, $\mathrm{Tyr}^{3}$-octreotide ( ${ }^{90} \mathrm{Y}$-DOTATOC). As a consequence of the replacement of phenylalanine by tyrosine as the third amino acid in the octapeptide, this analog has increased the affinity for somatostatin receptor subtype 2. The Basel group have used protocol with administration of a cumulative dose of $7.4 \mathrm{GBq} / \mathrm{m}^{2}$ in two cycles and showed partial remission in $7-28 \%$ of the patients and stable disease in $50 \%$ up to 
$80 \%$.[30-32] The responses were higher in pancreatic endocrine tumors than in carcinoid tumors. Dosimetric and dose-escalating phase I studies of ${ }^{90}$ Y-DOTATOC have been performed in Milan, Italy, both with and without the administration of renal-protecting agents.[33] Doses up to 5.6 GBq per cycle were administered. Responses were reported to be partial remission in about $29 \%$ of the patients. In a multicenter, phase I trial with ${ }^{90}$ Y-DOTATOC 60 patients received escalating doses of radioactivity without reaching the maximum tolerated single dose. The cumulative radiation dose to the kidneys was limited to 27 Gy. Concomitant amino-acid infusion was performed. Partial remission was obtained in 30\% and stable disease in 50\%.[34] In a large international multicenter trial ${ }^{90} \mathrm{Y}$-edotreotide was administered of 3 cycles of $4.4 \mathrm{GBq}$ in 90 patients, $75 \%$ showed stable disease or response.[35] Recently response, survival and long-term toxicity was evaluated after treatment with ${ }^{90}$ Y DOTATOC in 1.109 NET patients. Objective response was noted in 34\%, median survival 44.7 months over all $12 \%$ haematological grade $3 / 4$ toxicity was found and $9 \%$ grade $3 / 4$ renal toxicity.[36]

\section{Lu-DOTATATE}

${ }^{177} \mathrm{Lu}$-DOTA, $\mathrm{Tyr}^{3}$-octreotate represents the third generation of somatostatin receptor targeted radionuclide therapies and has been used in several hospitals since the beginning of year 2000. The only difference between DOTA, $\mathrm{Tyr}^{3}$-octreotate and DOTA, $\mathrm{Tyr}^{3}$-octreotide is that the C-terminal threoninol of DOTA, Tyr $^{3}$-octreotide is replaced with the amino acid, threonine. As a result, DOTATATE displays improved binding to somatostatin receptor positive tissues when compared with DOTATOC. Gastroenteropancreatic tumors predominantly express subtype 2 of the somatostatin receptor and DOTATATE has a six-fold to nine-fold increased affinity for this receptor subtype.[37, 38] ${ }^{177} \mathrm{Lu}-$ labeled somatostatin analogs have an important practical advantage over their ${ }^{90} Y$-labeled counterparts: ${ }^{177} \mathrm{Lu}$ is not a pure $\beta$ emitter, but also emits low-energy $\gamma$ rays, which allows direct post-therapy imaging and dosimetry.[39] In a study by Kwekkeboom and co-workers ${ }^{177} \mathrm{Lu}$ therapy was applied in a large group of patients.[40] The patients received up to an intended cumulative radioactive dose of 22.2-29.6 GBq limiting the dose to the kidneys to $23 \mathrm{~Gy}$. The treatment interval between the doses was 6-10 weeks applying 4 courses in most patients. 504 patients were treated according to the study protocol. Adverse effects within the first 24 hours were nausea in $25 \%$ of administrations, vomiting in $10 \%$ and pain in $10 \%$. Haematological adverse effects (WHO grades 3 or 4 ) occurred in $3.6 \%$. Mild and reversible alopecia was reported by $62 \%$ of the patients. Serous, delayed adverse effects occurred in 9 patients of whom 2 had decline in kidney function and 1 patient developed renal insufficiency 1.5 years after receiving her last treatment. Liver toxic effects occurred in 3 patients who had very extensive hepatic metastases. Tumors size was evaluated in 310 patients and partial response was obtained in $28 \%$, stable disease in $51 \%$, and progressive disease in $20 \%$. Median time to progression was 40 months. Median overall survival after initiation of treatment was 46 months. The most important predictive factor for survival was treatment outcome progression versus non-progressive disease. Other predictive factors included extent of liver metastases, a low Karnofsky performance status score, baseline weight loss, and to a lesser degree presence of bone metastases. A survival benefit for patients treated with ${ }^{177} \mathrm{Lu}$-octreotate was 23-69 months in comparisons with historical controls. Quality of life was also evaluated in 50 patients using the Cancer Quality-of-Life Questionnaire C30. Global health status and quality of life markedly improved after therapy with ${ }^{177} \mathrm{Lu}$-octreotate.[41] Our group has treated 300 patients with the same protocol as the Rotterdam group with similar response rates (OR 42\%). Patients with rectal carcinoids show the best responses (Figure 8).

\section{Other Radiolabeled Somatostatin Analogs}

Lanreotide can be labeled with ${ }^{111}$ In for diagnostic and therapeutic purposes and with ${ }^{90} \mathrm{Y}$ for therapeutic use.[42] Use of radiolabeled lanreotide has been advocated because of its increased affinity for somatostatin receptor subtypes 3 and 4 when compared with 111In-octreotide, but this claim is questionable.[37] Although radiolabeled lanreotide has been used to treat patients with NETs the affinity of this agent is less than that of radiolabeled DOTATOC or Octreotate for somatostatin receptor subtype 2, which is over-expressed in this class of tumors.[38]

DOTANOC is a peptide in which the third amino acid of octreotide (phenylalanine) is replaced by 1-naphthylalanine. DOTANOC has a good affinity for somatostatin receptor subtypes 2, 3 and 5 which might be important for imaging and treatment of tumors with atypical expression of these receptor subtypes.[43, 44] 


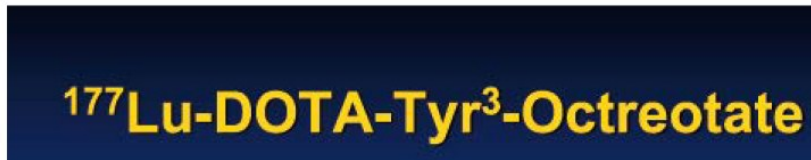

\section{Effect of therapy over time}
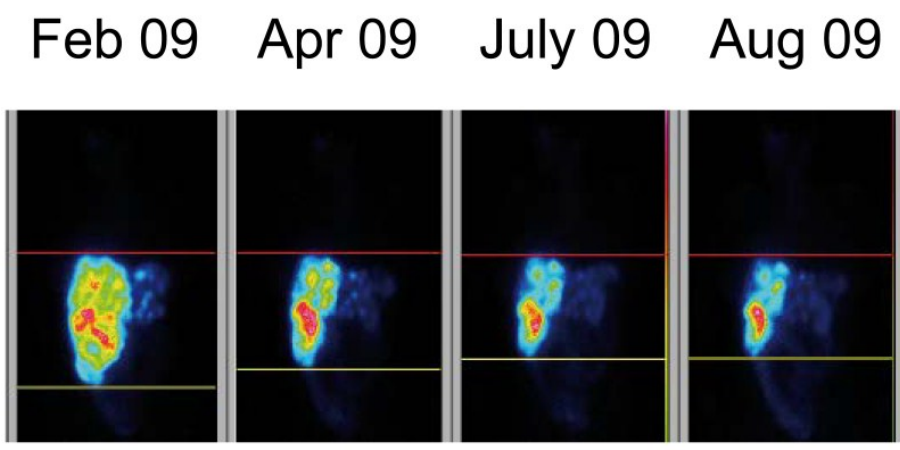

Jan 10

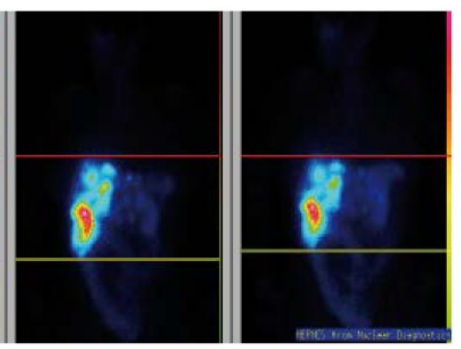

With courtesy from Ulrike Garske

Figure 8. Treatment with ${ }^{177}$ Lutetium-DOTA, $\mathrm{Tyr}^{3}$-octreotate in a patient with rectal carcinoid demonstrating effect of treatment over time.

\section{Summary}

PRRT with radiolabeled somatostatin analogs is a promising new option for these individuals provided that tumoral uptake of these agents is adequate on pre-treatment SRS (grade 3 and grade 4). Treatment with any of the various labeled somatostatin analogs can result in symptomatic improvement but reduction of tumor size is seldom achieved with Indium-labeled somatostatin analogs. Large variation exists in the reported anti-tumor effect of ${ }^{90}$ Y-DOTATOC between various studies. But an objective response was achieved in 9-33\% of patients. ${ }^{177} \mathrm{Lu}$-DOTATATE objective responses have been achieved in about $40 \%$ of patients; stable disease was present in $35-50 \%$. Adverse effects of PRRT are few and mostly mild, certainly when renal-protective agents are used. Serious, delayed adverse effects such as myelodysplastic syndrome, renal failure or leukemia are noted. The median duration of responses to 
therapy with ${ }^{90} \mathrm{Y}$-DOTATOC and ${ }^{177} \mathrm{Lu}$-DOTATATE is 30 months and 40 months, respectively. Large randomized clinical trials of PRRT are still needed. Another item which has to be evaluated is the value of dosimetry. Nevertheless, PRRT might soon become an important therapy for patients with metastatic or inoperable GEP-NETs.[45]

\section{Competing Interests}

The authors have declared that no competing interest exists.

\section{References}

1. Lawrence B, Gustafsson BI, Chan A, Svejda B, Kidd M, Modlin IM. The epidemiology of gastroenteropancreatic neuroendocrine tumors. Endocrinol Metab Clin North Am. 2011; 40: 1-18.

2. Modlin IM, Oberg K, Chung DC, Jensen RT, de Herder WW, Thakker RV, et al. Gastroenteropancreatic neuroendocrine tumours. Lancet Oncol. 2008; 9: 61-72.

3. Pearse AG. The cytochemistry and ultrastructure of polypeptide hormone-producing cells of the APUD series and the embryologic, physiologic and pathologic implications of the concept. J Histochem Cytochem. 1969; 17: 303-13.

4. Reubi JC, Waser B. Concomitant expression of several peptide receptors in neuroendocrine tumours: molecular basis for in vivo multireceptor tumour targeting. Eur J Nucl Med Mol Imaging. 2003; 30: 781-93.

5. Lee DY, Li KC. Molecular theranostics: a primer for the imaging professional. AJR Am J Roentgenol. 2011; 197: 318-24.

6. Krenning EP, Bakker WH, Breeman WA, Koper JW, Kooij PP, Ausema L, et al. Localisation of endocrine-related tumours with radioiodinated analogue of somatostatin. Lancet. 1989; 1: 242-4.

7. Krenning EP, Bakker WH, Kooij PP, Breeman WA, Oei HY, de Jong $\mathrm{M}$, et al. Somatostatin receptor scintigraphy with indium-111-DTPA-D-Phe-1-octreotide in man: metabolism, dosimetry and comparison with iodine-123-Tyr-3-octreotide. J Nucl Med. 1992; 33: 652-8.

8. Krenning EP, Kwekkeboom DJ, Bakker WH, Breeman WA, Kooij $\mathrm{PP}$, Oei HY, et al. Somatostatin receptor scintigraphy with

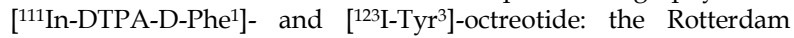
experience with more than 1000 patients. Eur J Nucl Med. 1993; 20: 716-31.

9. Jensen RT. Endocrine tumors of the gastrointestinal tract and pancreas. In: Kasper DL, Fauci AS, Longo DL, et al, editors. Harrison's principles of internal medicine. New Yrok: McGraw-Hill. 2005: 2221-31.

10. Kaltsas GA, Besser GM, Grossman AB. The diagnosis and medical management of advanced neuroendocrine tumors. Endocr Rev. 2004; 25: 458-511.

11. Koukouraki S, Strauss LG, Georgoulias V, Schuhmacher J, Haberkorn U, Karkavitsas N, et al. Evaluation of the pharmacokinetics of ${ }^{68} \mathrm{Ga}$-DOTATOC in patients with metastatic neuroendocrine tumours scheduled for ${ }^{90} \mathrm{Y}$-DOTATOC therapy. Eur J Nucl Med Mol Imaging. 2006; 33: 460-6.

12. Kowalski J, Henze M, Schuhmacher J, Macke HR, Hofmann M, Haberkorn U. Evaluation of positron emission tomography imaging using $\left[{ }^{68} \mathrm{Ga}\right]-D O T A-D-P h e^{1}-\mathrm{Tyr}^{3}$-Octreotide in comparison to [111 In]-DTPAOC SPECT. First results in patients with neuroendocrine tumors. Mol Imaging Biol. 2003; 5: 42-8.

13. Baum RP, Prasad V. Molecular imaging of neuroendocrine tumors using Ga-68 DOTA-NOC receptor PET/CT semiquantitative analyses after 1,257 studies (EPOS). European Congress of Radiology. Vienna, Austria; 2007.

14. Antunes P, Ginj M, Zhang H, Waser B, Baum RP, Reubi JC, et al. Are radiogallium-labelled DOTA-conjugated somatostatin analogues superior to those labelled with other radiometals? Eur J Nucl Med Mol Imaging. 2007; 34: 982-93.
15. Gabriel M, Decristoforo C, Kendler D, Dobrozemsky G, Heute D, Uprimny $\mathrm{C}$, et al. ${ }^{68} \mathrm{Ga}$-DOTA-Tyr ${ }^{3}$-octreotide PET in neuroendocrine tumors: comparison with somatostatin receptor scintigraphy and CT. J Nucl Med. 2007; 48: 508-18.

16. Eriksson B, Bergstrom M, Orlefors H, Sundin A, Oberg K, Langstrom B. Use of PET in neuroendocrine tumors. In vivo applications and in vitro studies. Q J Nucl Med. 2000; 44: 68-76.

17. Adams S, Baum R, Rink T, Schumm-Drager PM, Usadel KH, Hor G. Limited value of fluorine-18 fluorodeoxyglucose positron emission tomography for the imaging of neuroendocrine tumours. Eur J Nucl Med. 1998; 25: 79-83.

18. Sundin A, Eriksson B, Bergstrom M, Langstrom B, Oberg K, Orlefors H. PET in the diagnosis of neuroendocrine tumors. Ann N Y Acad Sci. 2004; 1014: 246-57.

19. Binderup T, Knigge U, Loft A, Federspiel B, Kjaer A. 18F-fluorodeoxyglucose positron emission tomography predicts survival of patients with neuroendocrine tumors. Clin Cancer Res. 2010; 16: 978-85.

20. Gazdar AF, Helman LJ, Israel MA, Russell EK, Linnoila RI, Mulshine JL, et al. Expression of neuroendocrine cell markers L-dopa decarboxylase, chromogranin A, and dense core granules in human tumors of endocrine and nonendocrine origin. Cancer Res. 1988; 48: 4078-82.

21. Ahlstrom H, Eriksson B, Bergstrom M, Bjurling P, Langstrom B, Oberg K. Pancreatic neuroendocrine tumors: diagnosis with PET. Radiology. 1995; 195: 333-7.

22. Jacob T, Grahek D, Younsi N, Kerrou K, Aide N, Montravers F, et al. Positron emission tomography with $\left[{ }^{18} \mathrm{~F}\right] \mathrm{FDOPA}$ and $\left[{ }^{18} \mathrm{~F}\right] \mathrm{FDG}$ in the imaging of small cell lung carcinoma: preliminary results. Eur J Nucl Med Mol Imaging. 2003; 30: 1266-9.

23. Orlefors H, Sundin A, Ahlstrom H, Bjurling P, Bergstrom M, Lilja A, et al. Positron emission tomography with 5-hydroxytryprophan in neuroendocrine tumors. J Clin Oncol. 1998; 16: 2534-41.

24. Koopmans KP, Neels OC, Kema IP, Elsinga PH, Sluiter WJ, Vanghillewe $\mathrm{K}$, et al. Improved staging of patients with carcinoid and islet cell tumors with ${ }^{18}$ F-dihydroxy-phenyl-alanine and ${ }^{11} \mathrm{C}$-5-hydroxy-tryptophan positron emission tomography. J Clin Oncol. 2008; 26: 1489-95.

25. Rufini V, Calcagni ML, Baum RP. Imaging of neuroendocrine tumors. Semin Nucl Med. 2006; 36: 228-47.

26. Valkema R, De Jong $M$, Bakker WH, Breeman WA, Kooij PP, Lugtenburg PJ, et al. Phase I study of peptide receptor radionuclide therapy with [In-DTPA]octreotide: the Rotterdam experience. Semin Nucl Med. 2002; 32: 110-22.

27. Anthony LB, Woltering EA, Espenan GD, Cronin MD, Maloney TJ, McCarthy KE. Indium-111-pentetreotide prolongs survival in gastroenteropancreatic malignancies. Semin Nucl Med. 2002; 32: 123-32.

28. Buscombe JR, Caplin ME, Hilson AJ. Long-term efficacy of high-activity ${ }^{111}$ In-pentetreotide therapy in patients with disseminated neuroendocrine tumors. J Nucl Med. 2003; 44: 1-6.

29. Delpassand ES, Sims-Mourtada J, Saso H, Azhdarinia A, Ashoori F, Torabi F, et al. Safety and efficacy of radionuclide therapy with high-activity In-111 pentetreotide in patients with progressive neuroendocrine tumors. Cancer Biother Radiopharm. 2008; 23: 292-300.

30. Otte A, Herrmann R, Heppeler A, Behe M, Jermann E, Powell P, et al. Yttrium-90 DOTATOC: first clinical results. Eur J Nucl Med. 1999; 26: 1439-47.

31. Waldherr C, Pless M, Maecke HR, Haldemann A, Mueller-Brand J. The clinical value of [ ${ }^{90} \mathrm{Y}$-DOTA]-D-Phe1-Tyr ${ }^{3}$-octreotide ( ${ }^{90}$ Y-DOTATOC) in the treatment of neuroendocrine tumours: a clinical phase II study. Ann Oncol. 2001; 12: 941-5.

32. Waldherr C, Pless M, Maecke HR, Schumacher T, Crazzolara A, Nitzsche EU, et al. Tumor response and clinical benefit in neuroendocrine tumors after 7.4 GBq ${ }^{90}$ Y-DOTATOC. J Nucl Med. 2002; 43: 610-6.

33. Chinol M, Bodei L, Cremonesi M, Paganelli G. Receptor-mediated radiotherapy with Y-DOTA-DPhe-Tyr-octreotide: the experience of the European Institute of Oncology Group. Semin Nucl Med. 2002; 32: 141-7. 
34. Valkema R, Pauwels S, Kvols LK, Barone R, Jamar F, Bakker WH, et al. Survival and response after peptide receptor radionuclide therapy with $\left.{ }^{90} \mathrm{Y}-\mathrm{DOTA}{ }^{0}, \mathrm{Tyr}^{3}\right]$ octreotide in patients with advanced gastroenteropancreatic neuroendocrine tumors. Semin Nucl Med. 2006; 36: 147-56.

35. Bushnell DL, Jr., O'Dorisio TM, O'Dorisio MS, Menda Y, Hicks RJ, Van Cutsem E, et al. ${ }^{90}$ Y-edotreotide for metastatic carcinoid refractory to octreotide. J Clin Oncol. 2010; 28: 1652-9.

36. Imhof A, Brunner P, Marincek N, Briel M, Schindler C, Rasch H, et al. Response, survival, and long-term toxicity after therapy with the radiolabeled somatostatin analogue [ $\left.{ }^{90} \mathrm{Y}-\mathrm{DOTA}\right]-\mathrm{TOC}$ in metastasized neuroendocrine cancers. J Clin Oncol. 2011; 29: 2416-23.

37. Reubi JC, Schar JC, Waser B, Wenger S, Heppeler A, Schmitt JS, et al. Affinity profiles for human somatostatin receptor subtypes SST1-SST5 of somatostatin radiotracers selected for scintigraphic and radiotherapeutic use. Eur J Nucl Med. 2000; 27: 273-82.

38. Reubi JC, Waser B, Schaer JC, Laissue JA. Somatostatin receptor sst1-sst5 expression in normal and neoplastic human tissues using receptor autoradiography with subtype-selective ligands. Eur J Nucl Med. 2001; 28: 836-46.

39. Kwekkeboom DJ, Bakker WH, Kooij PP, Konijnenberg MW, Srinivasan A, Erion JL, et al. [ $\left.{ }^{177} \mathrm{Lu}-\mathrm{DOTA}^{0}, \mathrm{Tyr} 3\right]$ octreotate: comparison with ${ }^{111}$ In-DTPA ${ }^{0}$ octreotide in patients. Eur J Nucl Med. 2001; 28: 1319-25.

40. Kwekkeboom DJ, de Herder WW, Kam BL, van Eijck CH, van Essen M, Kooij PP, et al. Treatment with the radiolabeled somatostatin analog $\left[{ }^{177} \mathrm{Lu}-\mathrm{DOTA}{ }^{0}, \mathrm{Tyr}^{3}\right]$ octreotate: toxicity, efficacy, and survival. J Clin Oncol. 2008; 26: 2124-30.

41. Teunissen JJ, Kwekkeboom DJ, Krenning EP. Quality of life in patients with gastroenteropancreatic tumors treated with [177Lu-DOTA ${ }^{0}$, Tyr $\left.^{3}\right]$ octreotate. J Clin Oncol. 2004; 22: 2724-9.

42. Virgolini I, Britton K, Buscombe J, Moncayo R, Paganelli G, Riva P. In- and Y-DOTA-lanreotide: results and implications of the MAURITIUS trial. Semin Nucl Med. 2002; 32: 148-55.

43. Valkema R, et al. Peptide receptor scintigraphy (PRS) with In-177-DOTANOC and peptide receptor radionuclide therapy (PRRT) with Lu-177-DOTANOC [abstract]. J Nucl Med. 2007; 48: 394.

44. Wild D, Schmitt JS, Ginj M, Macke HR, Bernard BF, Krenning E, et al. DOTA-NOC, a high-affinity ligand of somatostatin receptor subtypes 2, 3 and 5 for labelling with various radiometals. Eur J Nucl Med Mol Imaging. 2003; 30: 1338-47.

45. Wehrmann C, Senftleben S, Zachert C, Muller D, Baum RP. Results of individual patient dosimetry in peptide receptor radionuclide therapy with ${ }^{177} \mathrm{Lu}$ DOTA-TATE and ${ }^{177} \mathrm{Lu}$ DOTA-NOC. Cancer Biother Radiopharm. 2007; 22: 406-16. 